\title{
Towards a Conversational Interface for Authoring Intelligent Virtual Characters
}

\author{
Xinyi Wang \\ Disney Research Zurich \\ Switzerland
}

\author{
Samuel S. Sohn \\ Rutgers University \\ U.S.A.
}

\author{
Mubbasir Kapadia \\ Rutgers University \\ U.S.A.
}

\section{KEYWORDS}

Conversational authoring interface; natural language interface; intelligent virtual agent

\section{ACM Reference format:}

Xinyi Wang, Samuel S. Sohn, and Mubbasir Kapadia. 2019. Towards a Conversational Interface for Authoring Intelligent Virtual Characters. In Proceedings of ACM International Conference on Intelligent Virtual Agents, Paris, France, July 2-5, 2019 (IVA '19), 3 pages.

https://doi.org/10.1145/3308532.3329431

\section{INTRODUCTION}

The collaboration between creatives and domain architects is crucial for bringing virtual characters to life. Domain architects are technical experts who are tasked with formally designing intelligent virtual characters' domain knowledge, which is a symbolic representation of knowledge that the character uses to reason over its interactions with other agents. In the context of this work, domain knowledge encompasses the mental modeling of the character. Although the creation of interactive narratives requires substantial engineering expertise, it is also necessary to pick the brains of writers, artists, and animators alike to give the characters a boost of peculiarities. This intrinsically collaborative and interdisciplinary process brings about the challenge of bridging different mindsets and workflows in an efficient and effective way.

The conventional authoring process for virtual characters is heavily driven by engineering needs (shown in Figure 1a). This process burdens creative authors with inconsistent and cumbersome tasks, leaving little room for imagination and improvisation. As the intelligent system goes through updates, creatives are forced to adjust to new tools and take on new tasks in order to satisfy demands for creative input. Inconsistency and the lack of formality result in ineffective communication, repetitive tasks, underused data, and consequently, content of compromised quality.

The design of this interface, CO-AUTHOR (COnversational AUTHORing interface), takes advantage of the power of natural language and facilitates an authoring experience that emphasizes the creative freedom offered by writing. We value the use of natural language because it is the most intuitive medium for storytelling. To simulate the creative writing experience, CO-AUTHOR guides the author through sessions where (s)he writes short, atomic interactive scenes involving the character and the user. In each session,

Permission to make digital or hard copies of part or all of this work for personal or classroom use is granted without fee provided that copies are not made or distributed for profit or commercial advantage and that copies bear this notice and the full citation on the first page. Copyrights for third-party components of this work must be honored.

For all other uses, contact the owner/author(s).

IVA '19, July 2-5, 2019, Paris, France

(C) 2019 Copyright held by the owner/author(s).

ACM ISBN 978-1-4503-6672-4/19/07 _\$15.00

https://doi.org/10.1145/3308532.3329431 the author describes the character's action, the consequence of the action, the reasoning behind it, a sample dialogue that accompanies this scene, etc. The data collected through these conversations is modularized in a "scene-based" format. As data accumulates, domain architects refer to this reservoir of creative content when programming a character's domain knowledge and dialogue model. It should be noted that CO-AUTHOR is agnostic to the embodiment of the character.

The contribution of this work includes: a proposition for a collaborative authoring workflow that makes use of CO-AUTHOR; a proof-ofconcept prototype of CO-AUTHOR; and an evaluation of the usability and effectiveness of the prototype in the context of the proposed collaborative authoring workflow.

\section{RELATED WORK}

The authoring challenge that this work hopes to address aligns with existing lines of inquiries which focus on the authoring of non-linear narratives. A major cause for the difficulty of authoring interactive narratives is their non-linear structure. Authoring tools with graphical interfaces empower the author by visualizing non-linear narrative content $[4,5,11]$. These visualizations tend to follow a graph-based structure, which is generally consistent with the symbolic structure of the target information space. Visual environments also have the benefits of transparency and clarity.

We can generalize content authoring as a knowledge acquisition problem for graph-based information. Jambalaya [14] is a proposed solution for this general problem that applies SHriMP [15] - a visualization technique for hierarchically structured information spaces-to a knowledge management environment that helps domain experts navigate through complex information hierarchies. SHriMP faces the challenge of managing visual complexity, but users have suggested either (a) hiding the arcs drawn between related components by default or (b) incorporating filtering mechanisms that hide irrelevant arcs in order to improve usability. This implies that hiding the complexity would afford better focus to users.

CO-AUTHOR can be considered as the total embodiment of this "focus," because it hides everything but the scene in progress. This implicitly discourages the user from thinking about the exponential branching of interactions and instead allows the user to concentrate on enriching deep interactions. In doing so, we challenge the assertion made by Perlin and Goldberg [10] that it is insufficient to provide an author with a "tool for scripting long linear sequences," and we explore the potential of an untapped approach. We posit that a natural language interface would more efficiently facilitate this than a graphical or scripting interface.

March22 [7] is a prototype that lies between graphical/scripting and natural language interfaces. Authors use plain language to write the script for a given scene and a programming language to transition between scenes based on a user's decision. This is accompanied 
by a visualization of the transitions between scenes. Our approach differs in that we incorporate neither a graphical nor a scripting interface. Other tangential natural language interfaces include Façade [9], which performs real-time story comprehension in a gaming environment, interpreting the player's natural language input to push forward the storyline. STAR [2] guides the user to write linear narratives and answer logical questions while extracting world knowledge and inference rules. Sanghrajka et al. [12] introduces LISA, which dynamically builds knowledge base while the author is writing. Building upon LISA, CARDINAL $[8,13]$ is a combination of the graphical and natural language approaches. It allows creatives to write movie scripts using natural language and automatically creates meaningful visualizations and instant 3D animated pre-visualization of the script as it is being written.

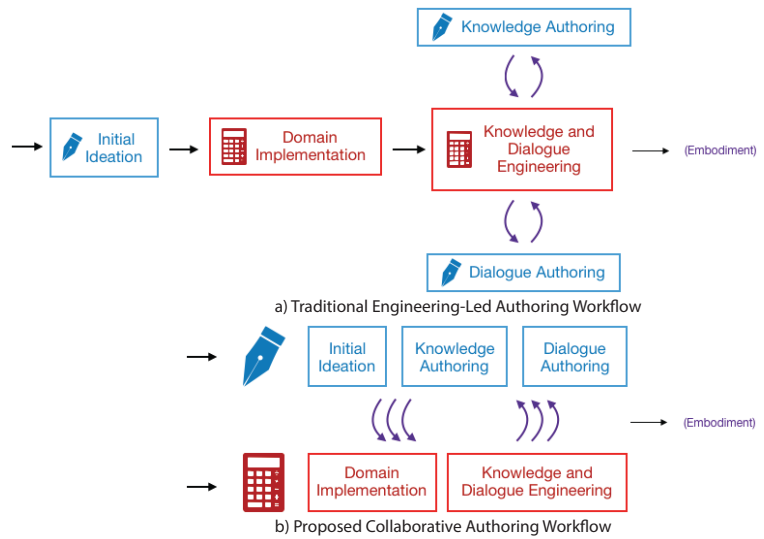

Figure 1: (a) Conventional authoring workflow which decouples creative tasks (blue), centering them around engineering tasks (red). (b) Proposed workflow which tightly couples all creative tasks and places them in parallel to engineering tasks.

\section{AUTHORING WORKFLOW}

Since the intelligent system is the most fundamental component of any intelligent virtual character, the authoring pipeline is heavily driven by engineering needs. Figure 1a provides a high-level visualization of this engineering-led authoring pipeline. The intuitive order of the authoring process goes from left to right. Through bluesky ideation, the author designs the story world and creates initial outlines for the character. This lays the foundation for developing a domain structure which stores the mechanisms that dictate how the virtual character acts emotionally, physically, and verbally in interactive narratives. Domain architects will then populate the domain with content provided by creative authors. Traditionally, the authoring of knowledge and dialogues are done separately. Depending on engineering needs, the author is often asked to provide content in multiple formats using a variety of tools. Lastly, behaviors and dialogues of the characters in the context of each narrative are embodied and performed in visual and audio means.

The main disadvantage of this workflow is the lack of balance between creative and engineering voices. As shown in Figure 1a, the central thread of the authoring pipeline is mostly led by engineering (in red). Creatives (in blue) are tasked to provide content that serves the system. To cater to the system's needs, they often need to switch between tools and platforms or use multiple at the same time. This process results in inefficient collaboration, cumbersome authoring experiences for creatives, and eventually, output of compromised quality.

\subsection{Proposed Collaborative Authoring Workflow}

While still considering the same set of tasks, we propose an alternative workflow which reorganizes them to orchestrate a more structured, fluent, and enjoyable collaborative authoring experience (Figure 1b). This workflow makes uses of CO-AUTHOR, a conversational interface which incorporates all tasks involving creative authors-initial ideation, knowledge authoring, and dialogue authoring-into a single platform.

Using this tool, the overall authoring pipeline becomes a fully collaborative cycle where creative authors and domain architects work in parallel. The creative content provided by authors is modularized such that each scene can be viewed as an independent entity. As the author accumulatively generates scenes, domain architects can work simultaneously and use the available creative content to build the character's domain knowledge and dialogue model. From each scene, meaningful information corresponding to different aspects of the character's domain knowledge-affordances, emotion rules, motivation rules - can be manually or automatically extracted.

\section{USER EXPERIENCE DESIGN}

Using CO-AUTHOR, the author goes through five question-answering dialogue segments in an authoring session. By posing questions in a defined sequence, this interface guides the author to think within confined scenarios. Despite the rigidness of the sequence, each question is designed to be open-ended and invites improvisation within the scope of each individual answer.

The prototype of CO-AUTHOR was created as a web application that operates on the browser. The visual graphic is generated using p5.js, a client-side Javascript library for graphic design [6]. The web backend uses the Node.js run-time environment [3].

Project Setup. The author is prompted to name the project and the main character of the scene. The input for character name will be used to generate more personalized questions in later interactions.

Character Setup. The interface asks the author for a general description of what the character is like. The author is allowed to submit multiple responses and end with the keyword "done."

State Extraction. The author is asked three questions regarding: the physical and emotional capabilities of the character; the physical and emotional capabilities of the user; and environmental factors. The goal of this segment is to obtain information about possible states in the world. We use a natural language processing module to parse the author's natural language input into states in the form of triplets: subject, verb, and prepositional complement. Through this process, we form a list of states - each containing a discrete possibility in the story world-from the author's natural language descriptions.

Scene Authoring. In this segment, the author is guided to describe a scene by answering a series of questions. Scene Setting has a multiple choice question where the author is asked to choose one or more from the states extracted from the previous segment in order to set up the beginning of a scene. Although this abstract state representation is an outlier within the overall interaction, which is in natural language, we elected to use this format to present a clear 
view of all discrete possibilities in the story world. In the Action sub-segment, the author describes what the character would do in this situation. The next sub-segments ask the author to describe the reasoning behind, consequence of, and emotional impact of the character's action. In the Interactivity sub-segment, the author indicates whether this interaction has a dialogue component to it, and if so, the interface asks the author to compose a short dialogue. Finish or New Scene. After the scene is finished, the author has the choice of either saving progress and exiting, or starting a new scene by returning to Scene Authoring.

\section{USER STUDY AND EVALUATIONS}

We performed user testing to evaluate the usability and effectiveness of the conversational interface. We performed the same test across 14 subjects within the age range 18-35 from the Greater Los Angeles area. Five subjects were familiar with the problem space of virtual character authoring, while the other nine were introduced to it for the first time. All subjects used the same desktop computer in the same location and environment for the tests. Every testing session consisted of two rounds: one structured and one unstructured. In the first structured round, all subjects were introduced to CO-AUTHOR for the first time and they were all instructed to author the same character. The purpose of this round of structured testing was to evaluate the usability and learnability of the tool, and to get the subjects' initial responses to the user experience.

\begin{tabular}{c|c} 
Question Measure & Average Agreement (0-4) \\
\hline Redundancy & 1.14 \\
Unclearness & 1.43 \\
Incompleteness & 1.64
\end{tabular}

Table 1: This table shows average scores from the first round of testing across 14 subjects, which all fall between "disagree" and "neutral."

For the first round, the 14 subjects scored CO-AUTHOR using the System Usability Scale. Based on a mapping of scaled SUS scores to common adjectives [1], the mean and median scores (81.07 and 82.5) fell in the range of "good" and "excellent," implying that the tool is both highly usable and learnable. Table 1 reports agreement scores which indicate that on average, the questions asked by CO-AUTHOR are neither redundant nor unclear for users and that they are comprehensive.

In the second unstructured round, as the subjects were more familiar with the tool, their task became authoring a fictional character of their own choice. The goal was to evaluate whether the tool enables the authoring of diverse characters, provides enjoyment, and encourages creativity. Most subjects agreed that they were able to create multiple characters with distinct personalities using CO-AUTHOR, giving an average score of 3.43/4.0 for character generalizability (between "agree" and "strongly agree"). According to the subjects' feedback, even though the interaction is designed as a conversation, it is still highly scaffolded and repetitive, somewhat hindering enjoyment and creative freedom. CO-AUTHOR allows the author to write freely when answering individual questions but does not provide higher-level autonomy over each scene or the authoring experience as a whole.

Based on qualitative and quantitative analyses over the questionnaire results, we find that the tool offers a favorable user experience and gives authors the ability to create a variety of unique virtual characters.

\section{CONCLUSIONS}

This work demonstrates an efficient means to orchestrate a fluent conversational authoring experience that amplifies creative voices in the engineering-oriented authoring pipeline for intelligent virtual characters. In order to take the leap from utility-based conversational agents to unique, interesting characters that bring immersion into narrative experiences, it is crucial that input from creative talent is properly gathered and employed in the overall authoring process. The design of CO-AUTHOR is rooted in the belief that human creativity can be better incorporated into intelligent virtual characters by using the appropriate interface, which we have hypothesized is conversational. With the proof-of-concept prototype, we have demonstrated the value of a conversational interface in facilitating an author-centric workflow.

\section{ACKNOWLEDGEMENTS}

This work has been funded in part by NSF IIS-1703883 and NSF SAS-1723869.

\section{REFERENCES}

[1] Aaron Bangor, Philip Kortum, and James Miller. 2009. Determining What Individual SUS Scores Mean: Adding an Adjective Rating Scale. J. Usability Studies 4, 3 (May 2009), 114-123. http://dl.acm.org/citation.cfm?id=2835587.2835589

[2] Irene-Anna Diakidoy, Antonis Kakas, Loizos Michael, and Rob Miller. 2015. STAR: A system of argumentation for story comprehension and beyond. In Proceedings of the 12th International Symposium on Logical Formalizations of Commonsense Reasoning. 64-70.

[3] Node.js Foundation. 2018. Node.js. (2018). https://nodejs.org/en/

[4] Daniel Fu and Ryan T. Houlette. 2002. Putting AI in Entertainment: An AI Authoring Tool for Simulation and Games. IEEE Intelligent Systems 17 (2002), $81-84$.

[5] Mubbasir Kapadia, Seth Frey, Alexander Shoulson, Robert W. Sumner, and Markus H. Gross. 2016. CANVAS: computer-assisted narrative animation synthesis. In Symposium on Computer Animation.

[6] NYU ITP et al. Lauren McCarthy, Processing Foundation. 2018. P5.js. (2018). https://p5js.org/

[7] Samuel Lynch, Charlie Hargood, and Fred Charles. 2017. Textual Authoring for Interactive Narrative. (2017).

[8] Marcel Marti, Jodok Vieli, Wojciech Witoń, Rushit Sanghrajka, Daniel Inversini, Diana Wotruba, Isabel Simo, Sasha Schriber, Mubbasir Kapadia, and Markus Gross. 2018. CARDINAL: Computer Assisted Authoring of Movie Scripts. In 23rd International Conference on Intelligent User Interfaces (IUI '18).

[9] Michael Mateas and Andrew Stern. 2003. Faąde: An Experiment in Building a Fully-Realized Interactive Drama. (04 2003).

[10] Ken Perlin and Athomas Goldberg. 1996. Improv: A System for Scripting Interactive Actors in Virtual Worlds. In Proceedings of the 23rd Annual Conference on Computer Graphics and Interactive Techniques (SIGGRAPH '96). ACM, New York, NY, USA, 205-216. https://doi.org/10.1145/237170.237258

[11] Mark O. Riedl and Andrew Stern. 2006. Believable Agents and Intelligent Story Adaptation for Interactive Storytelling. In Proceedings of the Third International Conference on Technologies for Interactive Digital Storytelling and Entertainment (TIDSE'06).

[12] Rushit Sanghrajka, Daniel Hidalgo, Patrick P Chen, and Mubbasir Kapadia. 2017. Lisa: Lexically intelligent story assistant. In Proceedings of the 13th Artificial Intelligence and Interactive Digital Entertainment Conference.

[13] Rushit Sanghrajka, Wojciech Witon, Sasha Schriber, Markus H. Gross, and Mubbasir Kapadia. 2018. Computer-Assisted Authoring for Natural Language Story Scripts. In AAAI. AAAI Press, 7811-7818.

[14] Margaret-Anne Storey, Mark Musen, John Silva, Casey Best, Neil Ernst, Ray Fergerson, and Natasha Noy. 2001. Jambalaya: Interactive visualization to enhance ontology authoring and knowledge acquisition in Protégé. In in Protégé. Workshop on Interactive Tools for Knowledge Capture K-CAP-2001.

[15] M.-A. D. Storey, K. Wong, F. D. Fracchia, and H. A. Mueller. 1997. On Integrating Visualization Techniques for Effective Software Exploration. In Proceedings of the 1997 IEEE Symposium on Information Visualization (InfoVis '97). 